\title{
Evaluation of Microvascular Patterns Alone Using Endocytoscopy with Narrow-Band Imaging for Diagnosing Gastric Cancer
}

\author{
Yusuke Horiuchi $^{a}$ Toshiaki Hirasawa ${ }^{a}$ Naoki Ishizuka ${ }^{b}$ Junki Tokura ${ }^{a}$ \\ Mitsuaki Ishioka ${ }^{a}$ Yoshitaka Tokai $^{a}$ Ken Namikawa ${ }^{a}$ Shoichi Yoshimizu ${ }^{a}$

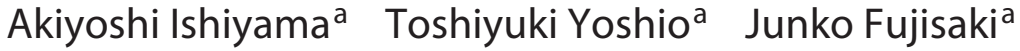 \\ aDepartment of Gastroenterology, Cancer Institute Hospital, Tokyo, Japan; bepartment of Clinical Trial Planning \\ and Management, Cancer Institute Hospital, Tokyo, Japan
}

\section{Keywords}

Diagnostic system · Gastric carcinoma - Microsurface

pattern · Magnifying endoscopy

\begin{abstract}
Introduction: Although endocytoscopy (EC) with narrowband imaging (NBI) is effective in diagnosing gastric cancer, no diagnostic system has been validated. We explored a specific diagnostic system for gastric cancer using EC with NBI. Methods: Equal numbers of images from cancerous and noncancerous areas (114 images each) were assessed by endoscopists with (development group: 33) and without (validation group: 28) specific training in magnifying endoscopy with NBI. Microvascular and microsurface patterns (MS) in each image were evaluated. Lesions were diagnosed as cancerous when patterns were deemed "irregular." The accuracy, sensitivity, specificity, positive predictive value, and negative predictive value of a diagnosis according to patterns on EC with NBI (microvascular pattern [MV] alone, MS alone, and both) were evaluated and compared between groups to determine the diagnostic performance. Results: In the development and validation groups, diagnoses based on the MV alone had significantly higher accuracy $(91.7 \%$ vs. $76.3 \%, p<$
\end{abstract}

karger@karger.com www.karger.com/dig

Karger $\stackrel{\text { ' }}{5}$

BOPEN ACCESS
(C) 2021 The Author(s)

Published by S. Karger AG, Basel

This is an Open Access article licensed under the Creative Commons Attribution-NonCommercial-4.0 International License (CC BY-NC) (http://www.karger.com/Services/OpenAccessLicense), applicable to the online version of the article only. Usage and distribution for commercial purposes requires written permission.
0.0001 and $92.5 \%$ vs. $67.5 \%, p<0.0001$, respectively) and sensitivity ( $88.6 \%$ vs. $68.3 \%, p<0.0001$ and $89.5 \%$ vs. $38.6 \%$, $p<0.0001$, respectively) than those based on the MS alone. In both groups, there were no significant differences in diagnostic accuracy between using the MV alone and both patterns. Discussion/Conclusion: Evaluation of the MV alone is a simple and accurate diagnostic method for gastric cancer. This system could find widespread applications in clinical practice.

(C) 2021 The Author(s) Published by S. Karger AG, Basel

\section{Introduction}

Gastric cancer is one of the most common causes of cancer-related deaths worldwide $[1,2]$. Recent advances in endoscopic equipment have led to increased detection rates for gastric cancer cases at early stages and decreased mortality rates [3-5]. The use of recently developed magnifying endoscopy (ME) with narrow-band imaging (NBI) (ME-NBI) in addition to conventional endoscopy has been reported to improve diagnostic performance in cases of gastric cancer $[6,7]$. The magnifying power of ME-NBI is approximately $\times 80-100$, which when com- 
bined with endocytoscopy (EC), a form of "ultra-magnifying endoscopy" [8-10], can yield a magnification of up to approximately $\times 400-500$; this level of magnification is similar to that of microscopy in pathological diagnosis. Therefore, the shape of cells and nuclei can be observed after staining with methylene blue and crystal violet. The usefulness of EC in evaluating various organs has been reported previously [8-15]; however, in the stomach, the presence of mucus can reduce the efficacy and accuracy of this method for diagnosing gastric cancer [13-15].

EC with NBI (EC-NBI) has been reported to be substantially more accurate than conventional ME-NBI in diagnosing colorectal cancer [16]. While there has been no report on the use of EC-NBI in diagnosing gastric cancer, we recently reported that EC-NBI exhibits a higher diagnostic performance for gastric cancer than does MENBI [17]. However, because a diagnostic system for ECNBI has not been established, its diagnostic performance was evaluated using the vessel plus surface (VS) classification $[7,18]$, which is the diagnostic system for ME-NBI. In contrast to ME-NBI, the EC-NBI method may differentiate between the cancerous and noncancerous areas in the stomach through an assessment of either the microvascular pattern (MV) or the microsurface pattern (MS) in each area. Nevertheless, such an ability has not been tested in practice because the use of EC-NBI is not widespread. Hence, this supplementary study aimed to explore this diagnostic system to expand the application of EC-NBI as a specific diagnostic method for gastric cancer.

\section{Materials and Methods}

This study is the post hoc analysis of a previous study [17]. The data of this post hoc analysis were newly calculated using the results already obtained from our previous retrospective, single-center observational study that compared the diagnostic performance of ME-NBI and that of EC-NBI [17]. Briefly, 118 lesions from 114 consecutive patients who had undergone endoscopic examination and endoscopic submucosal dissection (ESD) by 1 endoscopist from July 2016 to July 2019 were included. Case images and data were extracted from electronic medical records.

The inclusion criteria were as follows: (1) EC-NBI images were taken on the same day as treatment; and (2) EC-NBI images covered the outermost oral side of the cancerous area and adjacent noncancerous area to ensure that the boundary between the cancerous and noncancerous areas could be easily defined. The exclusion criteria were as follows: (1) unclear images due to mucus, blood, or halation; or (2) the presence of borderline lesions, such as adenomas.

Using the post-ESD pathological results as the gold standard, the cancerous and noncancerous areas were confirmed in all cases in accordance with the guidelines for gastric cancer treatment [19].
One instructor from the Japan Gastroenterological Endoscopy Society (JSGE) selected all the images. Additionally, another instructor from the JSGE confirmed whether all images satisfied the inclusion and exclusion criteria. GIF-Y0002 and GIF-H290EC endocytoscopes (Olympus Medical Systems, Tokyo, Japan) were used for EC-NBI.

\section{Diagnostic Method and Endoscopists}

A total of 61 endoscopists from 45 institutions, with different facilities, in Japan, participated in the diagnosis. Of these participating endoscopists, 33 had specialized training in ME-NBI, whereas 28 did not. Briefly, the background of endoscopists is as follows: $\geq 10$ years of experience with endoscopy, $65.6 \%$; experience with $\geq 10,000$ cases of endoscopy, $50.8 \%$; qualified as specialists by the Japanese Gastrointestinal Endoscopy Society, 68.9\%; specialized training in ME-NBI, $54.1 \%$; $\geq 5$ years of experience with ME-NBI, 34.4\%; and experience with EC-NBI, 13.1\% [17].

The diagnostic method applied was the VS classification (a diagnostic system for ME-NBI) proposed by the JSGE as a diagnostic guideline $[7,18]$, wherein each endoscopist determined whether the pattern in each image was regular, irregular, absent, or inconclusive. Lesions were diagnosed as cancerous when either the MV or MS was "irregular." Any other finding was considered noncancerous. The MV and MS on EC-NBI as per the VS classification are shown in Figure 1. In the EC-NBI image, a white line can be seen in addition to the MV. The white line has been reported to correspond to the gland lumen (crypt opening) in the diagnosis of colonic lesions [20]. Therefore, the evaluation of MS was defined as evaluating the shape of the white lines (regular, irregular, absent, or inconclusive).

In the previous study, diagnostic results of cancerous or noncancerous areas recorded by each endoscopist were aggregated to calculate the diagnostic performance of all endoscopists [17]. In contrast, the diagnostic results (regular, irregular, absent, or inconclusive) of the MS and/or MV of each image were collected using the original data of the previous study [17], and the diagnostic performance of all images using the MS and MV was newly calculated in this study.

\section{Evaluation Criteria}

Endoscopists with training in ME-NBI at specialized facilities reportedly had a higher diagnostic performance in ME-NBI than do those without specialized training [21]. The VS classification, which is a diagnostic system for ME-NBI, was established based on diagnoses by endoscopists with specialized training in ME-NBI [7]. Therefore, those endoscopists who participated in this study were divided into 2 groups based on whether they had received specialized training in ME-NBI: endoscopists with specialized training in ME-NBI were assigned to the development group, whereas those without specialized training in ME-NBI were allocated to the validation group. We explored and evaluated the diagnostic performance in both the development group and validation group to confirm whether the developed diagnostic system could be generalized to all endoscopists.

For each image, the diagnosis response by the maximum number of endoscopists was considered the final diagnosis (regular, irregular, absent, or inconclusive) for that image. If $>1$ response ranked highly, the final diagnosis for that image was deemed inconclusive. In contrast, if the endoscopists' diagnostic agreement rate for each image was low, the reliability of diagnosis was regard- 


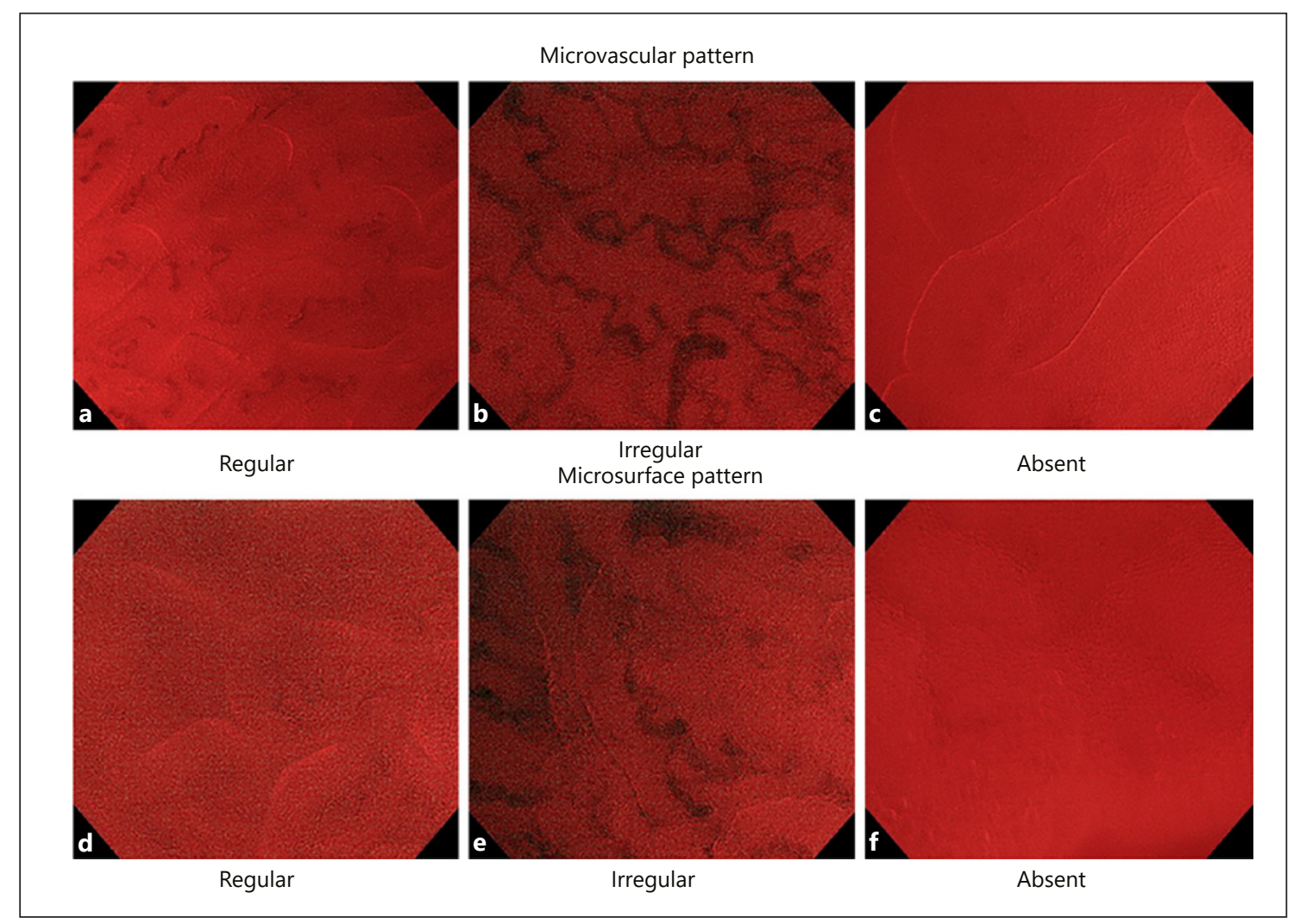

Fig. 1. Microvascular and MS classification (VS classification) based on endocytoscopy with NBI. If either the MV or the MS is "irregular," lesions are diagnosed as cancers. In this study, we evaluated endocytoscopy with NBI by applying this classification. The details of the classification are as follows: MV: a uniform blood vessels (regular). b Blood vessels that expand locally and have dif- ferent caliber meander (irregular). c No vascular findings (absent). MS (form of white line): $\mathbf{d}$ uniform surface structure (regular). e Nonuniform surface structure (irregular). $\mathbf{f}$ No surface structure (absent). VS, vessel plus surface; NBI, narrow-band imaging; MS, microsurface pattern; MV, microvascular pattern. ed as low, and generalization was considered difficult. Therefore, it was important to calculate the diagnostic agreement rate. We dealt with this as follows. The diagnostic agreement rate for each image was defined as the ratio of the maximum number of responses to all responses. The median diagnostic agreement rate was calculated for all images and was confirmed by the MV and MS in the development and validation groups. In the development and validation groups, the diagnoses of the MV and MS were aggregated for each image of cancerous and noncancerous lesions in terms of diagnostic agreement rate.

Based on the obtained aggregated results, the accuracy, sensitivity, specificity, positive predictive value (PPV), and negative predictive value (NPV) were calculated based on the patterns: MV alone (if only an irregular pattern was observed, the image indicated cancer), MS alone (if only an irregular pattern was observed, the image indicated cancer), and both patterns (if any of the patterns were irregular, the image indicated cancer) were determined. In both the development group and the validation group, diagnoses based on the patterns (MV alone, MS alone, and both) were compared. The diagnostic system with the fewest diagnostic items and highest diagnostic accuracy was regarded as the simplest and most accurate diagnostic system. We defined accuracy as follows: (number of correctly diagnosed cancerous lesions among actual cancerous lesions + number of correctly diagnosed noncancerous tissues among actual noncancerous tissues)/total number of images.

Subsequently, in the development group, cases in which the cancerous and noncancerous lesions were misdiagnosed were extracted using the simplest diagnostic system with the highest diagnostic accuracy. Characteristics were compared between correctly diagnosed cases and misdiagnosed cases. In images with cancerous lesions, we compared age, sex, location, macroscopic type, tumor diameter, invasion depth, ulcerative findings, histological type, and history of Helicobacter pylori infection, whereas in images with noncancerous lesions, age, sex, site, and history of $H$. pylori infection were compared. The cutoff values for age and tumor diameter were determined with reference to the overall median.

$H$. pylori-uninfected cases were defined as those that met the following 6 criteria: (1) absence of prior $H$. pylori eradication; (2) negative urea breath test results (UBiT; Otsuka, Tokushima, Japan); (3) negative $H$. pylori antibody status ( $H$. pylori antibody I; EIKEN Chemical, Tokyo, Japan); (4) negative pepsinogen test re- 


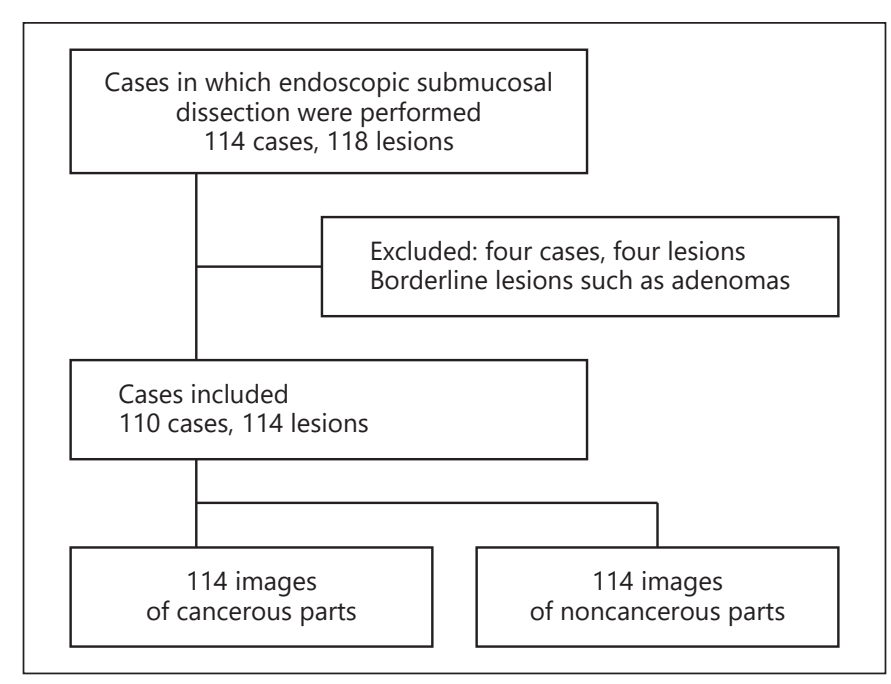

Fig. 2. Flow diagram showing the inclusion of patients.

sults (cutoff level for positive results: pepsinogen $\mathrm{I} \leq 70 \mathrm{ng} / \mathrm{mL}$ and pepsinogen I/II ratio $\leq 3$ ); (5) endoscopically confirmed positive regular arrangement of collecting venules in the lower gastric body [22]; and (6) histologically confirmed H. pylori-uninfected and negative inflammatory cell infiltration activity, as assessed by the updated Sydney System [23]. Cases that did not meet these criteria were designated as having a history of $H$. pylori infection.

The $H$. pylori-infected eradication group included (1) patients who were confirmed to be negative for $H$. pylori antibody (H. pylori antibody $\|$; EIKEN Chemical) during their first examination or those that had negative $\mathrm{C}$-urea breath test results (UBiT; Otsuka, Tokushima, Japan) after undergoing $H$. pylori eradication treatment at our hospital or in another hospital and (2) patients who were confirmed to have negative results for the urea breath test performed at least 4 weeks after the commencement of $H$. pylori eradication treatment if they had tested positive for $H$. pylori antibody or had positive results for the urea breath test performed during their first examination at our hospital. The $H$. pylori-infected noneradication group included patients who did not meet the abovementioned inclusion criteria.

\section{Statistical Analysis}

Medians with interquartile ranges (IQRs) were used when calculating the diagnostic agreement rate for all images. The accuracy, sensitivity, specificity, PPV, and NPV of diagnosis based on MV alone, diagnosis based on MS alone, and diagnosis based on both patterns were calculated along with the respective $95 \%$ confidence intervals. The McNemar's test was employed for accuracy, sensitivity, and specificity, whereas Fisher's exact test was used to evaluate PPV and NPV. The statistical significance threshold was set at $p<0.05 / 2$ with the Bonferroni method for the performance of 2 comparisons in the same population.

In a univariate analysis, Fisher's exact test was used for comparison between correctly diagnosed cases and misdiagnosed cases. For items with significant differences, logistic regression analysis was performed as multivariate analysis. Odds ratios and $95 \%$ confidence intervals were calculated, and the significance level was set at $<0.05$. Statistical analyses were performed using JMP version 13.2 software (SAS Institute, Cary, NC, USA).
Table 1. Patient characteristics

\begin{tabular}{|c|c|}
\hline Background & 110 cases, 114 lesions \\
\hline Age, years & $71(61.8-77)[26-87]$ \\
\hline Sex (male/female) & $74(67.3) / 36(32.7)$ \\
\hline \multicolumn{2}{|l|}{ Location } \\
\hline Upper third & $21(18.4)$ \\
\hline Middle third & $62(54.4)$ \\
\hline Lower third & $27(23.7)$ \\
\hline Gastric tube & $4(3.5)$ \\
\hline \multicolumn{2}{|l|}{ Macroscopic type } \\
\hline Elevated & $17(14.9)$ \\
\hline Flat & $6(5.3)$ \\
\hline Depressed & $88(77.2)$ \\
\hline Complex & $3(2.6)$ \\
\hline Tumor diameter, mm & $14(9-20.3)[1.5-57]$ \\
\hline \multicolumn{2}{|l|}{ Invasion depth } \\
\hline Intramucosal invasion (pT1a) & $95(83.3)$ \\
\hline \multicolumn{2}{|l|}{ Submucosal invasion (pT1b) } \\
\hline$<500 \mu \mathrm{m}$ & $15(13.2)$ \\
\hline$\geq 500 \mu \mathrm{m}$ & $4(3.5)$ \\
\hline \multicolumn{2}{|l|}{ Ulcerative findings } \\
\hline Present & $5(4.4)$ \\
\hline Absent & $109(95.6)$ \\
\hline \multicolumn{2}{|l|}{ Histological type } \\
\hline Differentiated & $90(78.9)$ \\
\hline Undifferentiated & $24(21.1)$ \\
\hline \multicolumn{2}{|c|}{ History of Helicobacter pylori infection } \\
\hline Infected & $105(92.1)$ \\
\hline Eradicated & $82(71.9)$ \\
\hline Not eradicated & $23(20.2)$ \\
\hline Uninfected & $9(7.9)$ \\
\hline
\end{tabular}

Data are presented as numbers (\%), except for age and tumor diameter, which are expressed as the median (IQR) [range]. IQR, interquartile range.

\section{Results}

\section{Inclusion of Patients and Their Background Data}

A total of 110 patients and 114 lesions met the inclusion criteria (shown in Fig. 2). A total of 228 images, divided equally between EC-NBI images of cancerous and noncancerous lesions, were used for diagnosis. The patients' background data are summarized in Table 1. The median age of the patients was 71 years (IQR: 61.8-77), and the median tumor diameter was $14 \mathrm{~mm}$ (IQR: 9-20.3 $\mathrm{mm})$.

\section{Diagnostic Agreement Rate for All Images}

Table 2 presents the diagnostic agreement rate for all images. In the development group, MV and MS were observed in $84.8 \%$ (IQR: 72.7-93.9\%) and 69.7\% (IQR: 
Table 2. Median diagnostic agreement rate for each finding in all images

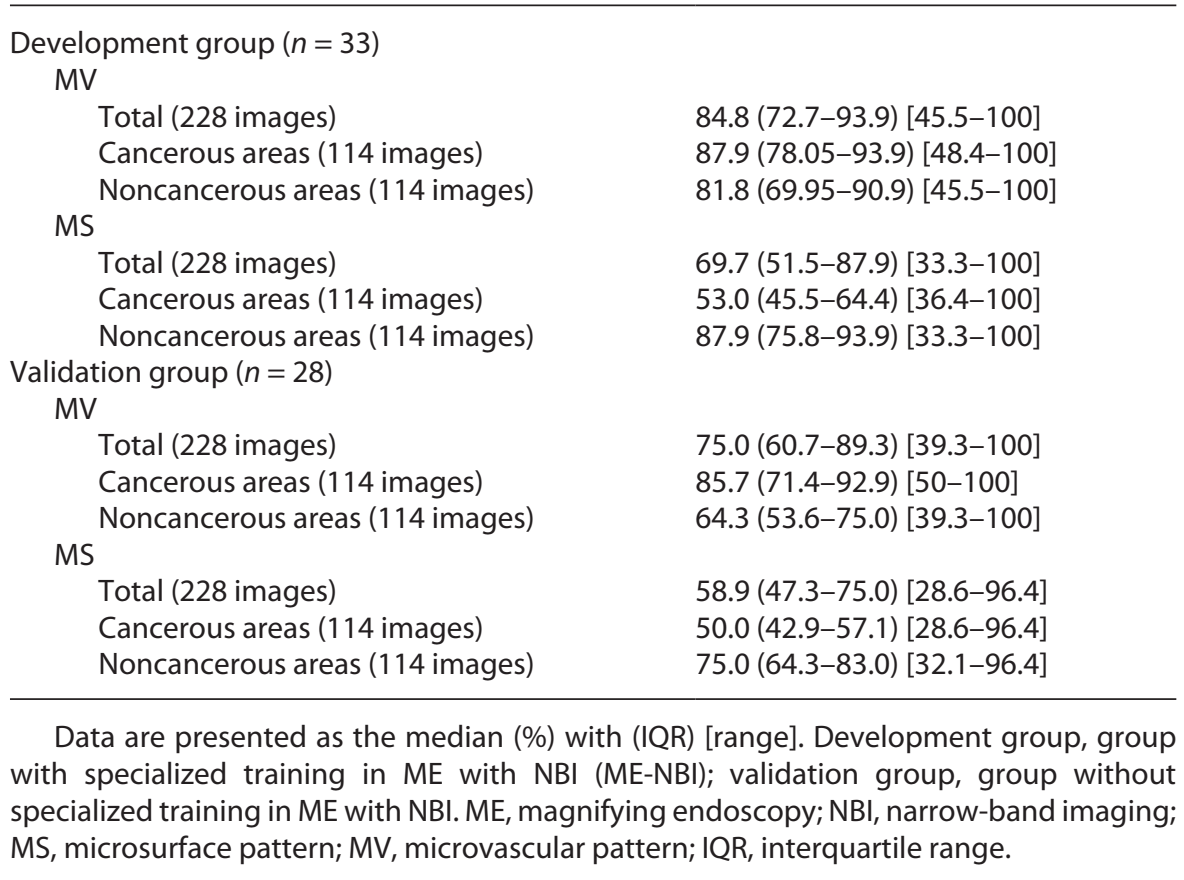

51.5-87.9\%) of all images, respectively. In the validation group, MV and MS were identified in 75.0\% (IQR: 60.789.3\%) and 58.9\% (IQR: 47.3-75.0\%) of all images, respectively. In terms of MV, the lower limit of the IQR was above $50 \%$ in both groups. However, with respect to MS, the lower limit of the IQR for the validation group was below $50 \%$.

\section{Diagnosis of $M V$ and $M S$}

Table 3 shows the diagnoses of MV and MS. In the development group, the combination of a MV regular pattern and MS regular pattern was found in $93.9 \%$ of the noncancerous areas. The combination of a MV irregular pattern and MS irregular pattern was found in $54.5 \%$ of the cancerous areas, while a MV irregular pattern was found in $87.8 \%$ of the cancerous areas. Similar results were obtained in the validation group.

Comparisons of Diagnostic Performance between $M V$ Alone and MS Alone and between MV Alone and

\section{Both Patterns}

Diagnostic performance was calculated based on the results presented in Table 3. Comparisons of diagnostic performance between MV alone and MS alone and between MV alone and both patterns were conducted (Table 4). In the development group, the accuracy, sensitivity, and NPV of diagnosis based on MV alone were sig- nificantly higher than those of diagnosis based on MS alone. Regarding the comparison between MV alone and both patterns, no significant differences were observed in all items. The results of the validation group were similar to those of the development group.

\section{Comparisons of Patient Characteristics between}

Correctly Diagnosed Cases and Misdiagnosed Cases

Based on Diagnosis Using Evaluation of MV Alone

We also compared patient characteristics between correctly diagnosed cases and misdiagnosed cases based on diagnosis using evaluation of MV alone in cancerous lesions in the development group (Table 5). Based on the results shown in Table 1, the cutoff values for age and tumor diameter were 70 years and $15 \mathrm{~mm}$, respectively. A univariate analysis revealed significant differences in macroscopic type and history of H. pylori infection. Multivariate analysis of these 2 items revealed that the odds ratio for the flat macroscopic type was 12.1 times that for the elevated macroscopic type, and the odds ratio for $H$. pylori-uninfected cases was 17.3 times that of $H$. pylori-infected cases. Six cases satisfied these conditions, and they all involved undifferentiated type of cancers (signet ring cell carcinomas). Images from the 6 cases are shown in Figure 3. There were no significant differences in noncancerous areas (online suppl. Table 1; see www.karger.com/doi/10.1159/000520276 for all online suppl. material). 
Table 3. Diagnosis of microvascular and MSs

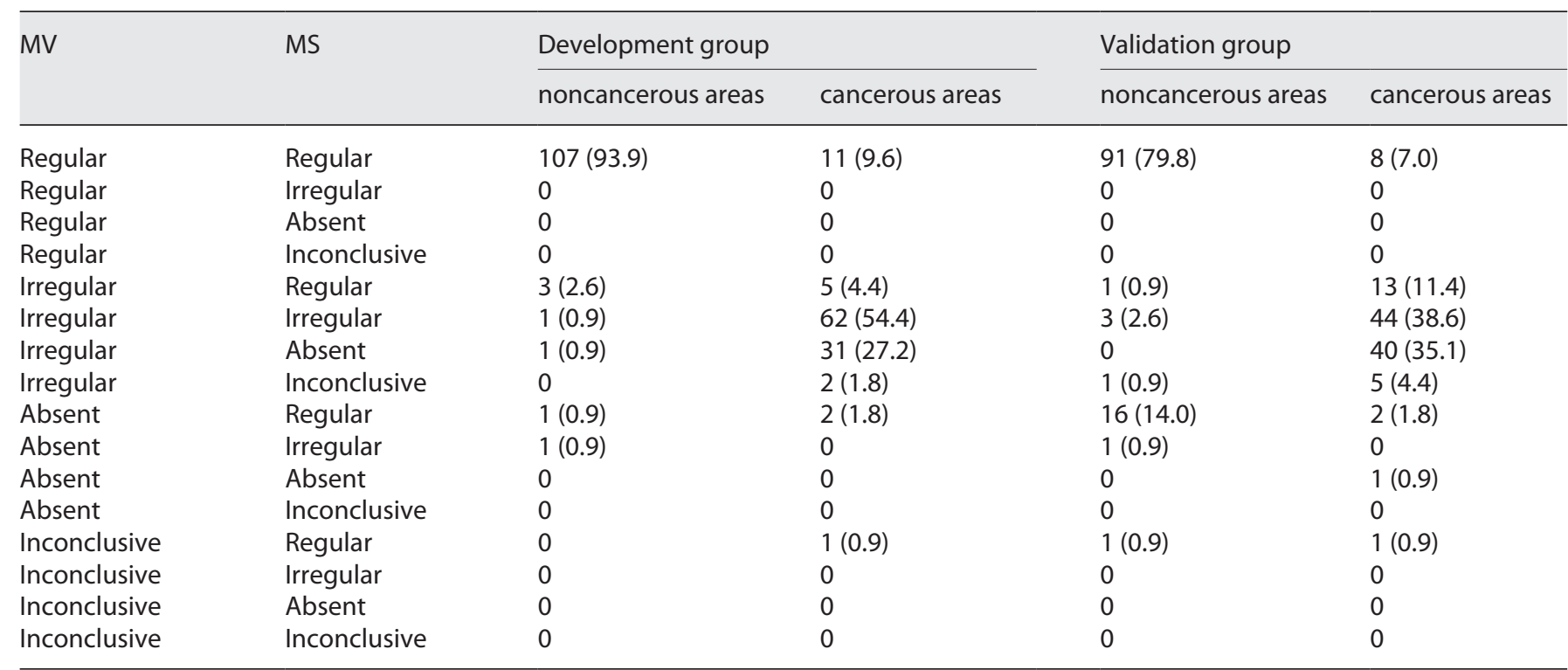

Data are presented as numbers (\%). MS, microsurface pattern; MV, microvascular pattern.

Table 4. Comparison of diagnostic performance between MV alone and MS alone and between MV alone and both MS and MV

\begin{tabular}{|c|c|c|c|c|c|}
\hline \multicolumn{6}{|c|}{ Development group } \\
\hline Accuracy & 91.7 (87.4-94.6) & $76.3(70.4-81.4)$ & $91.2(86.8-94.2)$ & $<0.0001$ & 0.3173 \\
\hline Sensitivity & 87.7 (80.4-92.5) & $54.4(45.2-63.2)$ & 87.7 (80.4-92.5) & $<0.0001$ & 1 \\
\hline Specificity & $95.6(90.1-98.1)$ & $98.3(93.8-99.5)$ & 94.7 (89.0-97.6) & 0.1797 & 0.3173 \\
\hline PPV & $95.2(89.3-97.9)$ & $96.9(89.3-99.1)$ & $94.3(88.2-97.4)$ & 0.7109 & 1 \\
\hline NPV & $88.6(81.8-93.1)$ & $68.3(60.8-74.9)$ & 88.5 (81.7-93.0) & $<0.0001$ & 1 \\
\hline Accuracy & 92.5 (88.4-95.3) & $67.5(61.2-73.3)$ & $92.1(87.9-94.9)$ & $<0.0001$ & 0.3173 \\
\hline Sensitivity & 89.5 (82.5-93.9) & $38.6(30.2-47.8)$ & 89.5 (82.5-93.9) & $<0.0001$ & 1 \\
\hline Specificity & $95.6(90.1-98.1)$ & $96.5(91.3-98.6)$ & $94.7(89.0-97.6)$ & 0.5637 & 0.3173 \\
\hline PPV & 95.3 (89.5-98.0) & 91.7 (80.4-96.7) & $94.4(88.4-97.4)$ & 0.4598 & 1 \\
\hline NPV & $90.1(83.5-94.2)$ & $61.1(53.8-67.9)$ & $90(83.3-94.2)$ & $<0.0001$ & 1 \\
\hline
\end{tabular}

Data are presented as percentage $(95 \% \mathrm{Cl})$. The statistical significance threshold was set at $p<0.05 / 2$ with the Bonferroni method for the performance of 2 comparisons in the same population. PPV, positive predictive value; NPV, negative predictive value; MS, microsurface pattern; MV, microvascular pattern; $\mathrm{Cl}$, confidence interval.

\section{Discussion/Conclusion}

To the best of our knowledge, this is the first study to propose a specific system for diagnosing gastric cancer using EC-NBI. Although this supplementary study was not initially planned, the data used in the post hoc analysis were newly calculated using the results already obtained by a previous study [17].
In both development group and validation group, the accuracy, sensitivity, and NPV of diagnoses using MV alone were significantly higher than those of diagnoses based on MS alone, and there were no significant differences in the comparison based on MV alone or both patterns. The gland lumen (MS in this study) varied depending on the strength of scope contact with the gastric mucosa and the method of light incident when observed by 
Table 5. Comparison of patient characteristics between correctly diagnosed cases and misdiagnosed cases based on MV alone in cancerous parts in the development group

\begin{tabular}{|c|c|c|c|c|c|}
\hline & \multicolumn{3}{|c|}{ Univariate analysis } & \multicolumn{2}{|c|}{ Multivariate analysis } \\
\hline & $\begin{array}{l}\text { misdiagnosis } \\
\text { (14) }\end{array}$ & $\begin{array}{l}\text { correct diagnosis } \\
(100)\end{array}$ & $p$ value & $\begin{array}{l}\text { odds ratio, } \\
95 \% \mathrm{Cl}\end{array}$ & $p$ value \\
\hline \multicolumn{6}{|l|}{ Age } \\
\hline$\geq 70$ years & $6(42.9)$ & $57(57)$ & 0.3942 & & \\
\hline$<70$ years & $8(57.1)$ & $43(43)$ & & & \\
\hline Sex (male) & $11(78.6)$ & $66(66)$ & 0.5433 & & \\
\hline \multicolumn{6}{|l|}{ Location } \\
\hline Upper third & $3(21.4)$ & $18(18)$ & 0.7458 & & \\
\hline Middle third & $6(42.9)$ & $56(56)$ & & & \\
\hline Lower third & $4(28.6)$ & $23(23)$ & & & \\
\hline Gastric tube & $1(7.1)$ & $3(3)$ & & & \\
\hline \multicolumn{6}{|l|}{ Macroscopic type } \\
\hline Elevated & $3(21.4)$ & $14(14)$ & 0.0003 & 1 (ref.) & \\
\hline Flat & $4(28.6)$ & $2(2)$ & & $12.1(1.4-108.0)$ & 0.0255 \\
\hline Depressed & $7(50.0)$ & $81(81)$ & & $2.9(0.6-14.1)$ & 0.1968 \\
\hline Complex & 0 & $3(3)$ & & $\mathrm{N} / \mathrm{A}$ & \\
\hline \multicolumn{6}{|l|}{ Tumor diameter } \\
\hline$\geq 15 \mathrm{~mm}$ & $4(28.6)$ & $49(49)$ & 0.252 & & \\
\hline$<15 \mathrm{~mm}$ & $10(71.4)$ & $51(51)$ & & & \\
\hline \multicolumn{6}{|l|}{ Invasion depth } \\
\hline Intramucosal invasion (pT1a) & $13(92.9)$ & $82(82)$ & 0.459 & & \\
\hline Submucosal invasion (pT1b) & $1(7.1)$ & $18(18)$ & & & \\
\hline \multicolumn{6}{|l|}{ Ulcerative findings } \\
\hline Present & $13(92.9)$ & $96(96)$ & 0.4872 & & \\
\hline Absent & $1(7.1)$ & $4(4)$ & & & \\
\hline \multicolumn{6}{|l|}{ Histological type } \\
\hline Differentiated & $8(57.1)$ & $82(82)$ & 0.0722 & & \\
\hline Undifferentiated & $6(42.9)$ & $18(18)$ & & & \\
\hline \multicolumn{6}{|c|}{ History of Helicobacter pylori infection } \\
\hline Infected & $8(57.1)$ & $97(97)$ & $<0.0001$ & 1 (ref.) & \\
\hline Not eradicated & 0 & $23(23)$ & & & \\
\hline Eradicated & $8(57.1)$ & $74(74)$ & & & \\
\hline Uninfected & $6(42.9)$ & $3(3)$ & & $17.3(3.1-96.0)$ & 0.0011 \\
\hline
\end{tabular}

Data are presented as numbers (\%) in univariate analysis. The statistical significance threshold was set at $p<$ 0.05 . $\mathrm{Cl}$, confidence interval; $\mathrm{N} / \mathrm{A}$, not available; $\mathrm{MV}$, microvascular pattern.

EC-NBI. Therefore, it is considered that there is a limit to the evaluation of the existence and morphology of the gland lumen using EC-NBI and that the evaluation of the MS is not stable. Therefore, diagnosis based on the MS is considered to have no significant effect on the diagnostic performance of both patterns, and thus, it can be omitted. We previously reported that EC-NBI showed higher gastric cancer diagnostic performance than did ME-NBI. As the VS classification was applied at that time, it was necessary to diagnose both patterns. If both MS and MV are required for diagnosis, taking multiple images is required until clear images are obtained for both MS and MV because taking only 1 image may result in clear MS and un- clear MV. If only MV is evaluated, the time required to obtain images with clear MS can be omitted. Therefore, the examination time is shorter than that of ME-NBI, the current standard test. Thus, compared to ME-NBI, the use of EC-NBI in clinical practice can simplify the diagnosis and shorten the examination time while maintaining the same high performance as with a diagnosis based on the VS classification, and the use of EC-NBI could be generalized.

The diagnostic agreement rate of MV was over $80 \%$ in all images in the development group, and $>70 \%$ in the validation group. However, with respect to MS, the lower limit of the IQR for the validation group was below $50 \%$. 

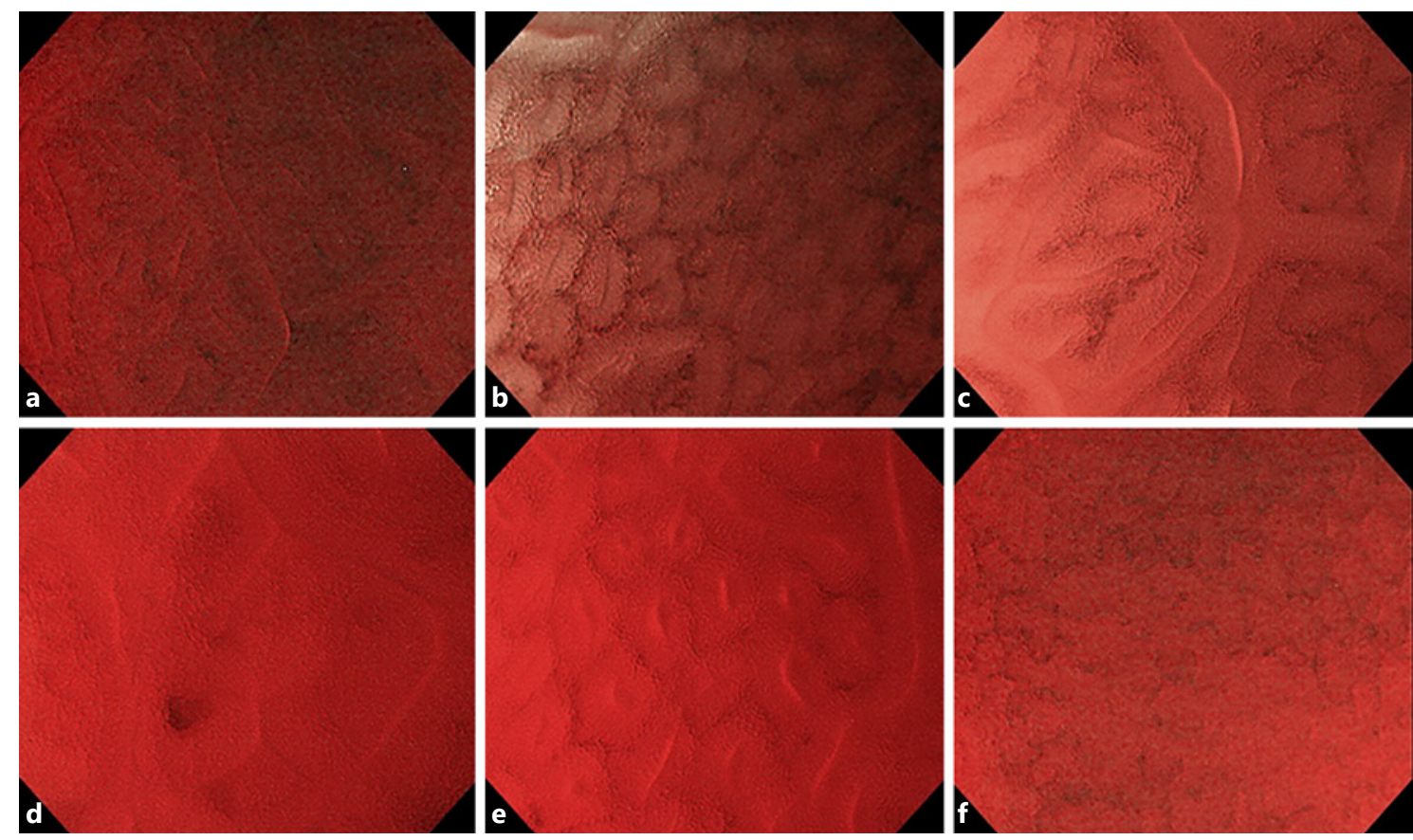

Fig. 3. Images from 6 cases of misdiagnosis using EC with NBI. The irregular MV was not recognized by the endoscopist in a-f, even though they are images of cancerous areas. EC, endocytoscopy; NBI, narrow-band imaging; $\mathrm{MV}$, microvascular pattern.

Thus, evaluating MV alone had a high diagnostic agreement rate among most endoscopists while evaluating MS varied with each endoscopist. Therefore, in clinical practice, while the evaluation of MS is expected to vary according to individual endoscopists, MV evaluation is expected to be reliable. Thus, evaluating MV alone appears to be appropriate for generalization.

In the comparison between misdiagnosed cases and correctly diagnosed cases involving cancerous lesions, a significantly larger number of cases showed the flat macroscopic type (all undifferentiated-type lesions), and most patients did not have a history of $H$. pylori infection (all undifferentiated-type lesions). Most undifferentiated-type cancers in patients not infected with $H$. pylori were found in the middle layer of the mucosa, with cancers not being exposed on the surface layer of the mucosa [24]. The VS classification reports about absent or regular MV or MS and rules out the diagnosis of cancer. This is the limitation of the VS classification system in diagnosing undifferentiated-type cancer $[7,18]$. EC-NBI, as well as ME-NBI, is a modality that can be used to observe the surface layer. Therefore, in EC-NBI as well as in ME-NBI, these cancer types are also not easily diagnosed using $\mathrm{MV}$ alone due to the inherent difficulties in recognizing MV.
In addition, these cases were misdiagnosed even with an evaluation of MS.

In contrast, in $H$. pylori-uninfected undifferentiatedtype cancers (mainly signet ring cell carcinomas), the difference in the surface structure size from the background normal mucosa on low magnification with NBI can be useful in making a diagnosis of gastric cancer, as previously reported [25]. Therefore, in clinical practice, we might be able to diagnose these cases using an endoscope equipped with EC-NBI because we can perform low magnification with NBI.

This study has some limitations. First, it was a retrospective single-center observational study. Second, borderline lesions were excluded. Third, as the endoscopists did not actually obtain the images themselves, it remains unknown whether images of similar quality to those in this study can be obtained in actual clinical practice. Finally, diagnosis was not performed at the time of realtime endoscopy in this study; the images were taken from all cases in which ESD was performed. This study also did not include cases in which gastric cancer was overlooked and ESD was not performed.

This study determined the diagnostic performance of several endoscopists using information from consecutive 
cases managed by 1 doctor in a hospital specializing in cancer treatment. However, diagnostic performance was evaluated for the majority of the 61 endoscopists from 45 facilities in Japan who participated in this study. Hence, we do not expect our findings to change considerably, even if the endoscopists change. In addition, because the study was designed to distinguish between cancerous and adjacent noncancerous areas, the usefulness of EC-NBI in differentiating borderline lesions from cancers could not be shown. However, the possibility of distinguishing adjacent noncancerous areas from gastric cancers was shown; therefore, EC-NBI may be considered useful for differentiating gastric cancers. The validation group showed almost the same diagnostic performance as the development group. This means that even endoscopists without specialized training in ME-NBI can achieve high diagnostic performance with EC-NBI.

As we previously reported, although it is necessary to use a soft hood to observe the target lesion at a distance with ME-NBI, it is possible to observe the target lesion closely with EC-NBI, without the need for a soft hood, by placing the tip of the scope in contact with the mucosa [17]. Therefore, respiratory fluctuations and body movements are less susceptible, and EC-NBI is simpler to operate than ME-NBI and able to perform without an advanced image capturing skill. Bleeding due to contact with the soft hood can also be avoided. Indeed, 8 lesions were excluded from the study due to inadequate ME-NBI, and none were excluded due to inadequate EC-NBI [17]. Therefore, EC-NBI may be useful in cancer screening, where sedation is often not used. Thus, we believe that EC-NBI is practical to all endoscopists for determining differentiation of gastric cancers. Therefore, despite the limitations, the results of this study are considered clinically meaningful at this stage.

In future, to eliminate these limitations, we plan to conduct a multicenter collaborative prospective trial to determine the diagnostic performance of evaluating MV alone for gastric tumors (including borderline lesions such as adenomas) by using EC-NBI and to confirm its utility in clinical practice. This study is useful in clinical practice because it provides valuable basic data for planning new trials.

In conclusion, evaluation of MV alone uses EC-NBI as a simple diagnostic system for gastric cancer showed high agreement rates and high performance. This result suggests that evaluating MV alone using EC-NBI can be expanded for the diagnosis of gastric cancer in clinical practice. We hope that this study will be the first step toward the worldwide application of EC-NBI.

Evaluation Microvascular Patterns

Endocytoscopy

\section{Acknowledgments}

The authors thank the 61 endoscopists from the 45 institutions for evaluating the images.

\section{Statement of Ethics}

This study was conducted in accordance with the principles outlined in the Declaration of Helsinki and its later amendments and was approved by the Institutional Review Board (IRB) of the Cancer Institute Hospital, Tokyo (Approval No. 2019-1032). All identifying personal information was removed while recording the data used in this study. Written informed consent for the use of pathological specimens and imaging data for research purposes were obtained from each patient in a previous study [17]. Written informed consent for participating in the study was waived by the IRB of the Cancer Institute Hospital, Tokyo, due to the retrospective nature of this study.

\section{Conflict of Interest Statement}

Yusuke Horiuchi has received a research grant from Grant-inAid for Early-Career Scientists (21K15962) and personal fees for specific speaking and teaching commitments as honoraria from Olympus Corporation, Tokyo, Japan and Kaken Pharmaceutical Co., Ltd, Tokyo, Japan. Toshiyuki Yoshio has received research grants from the Takeda Science Foundation and a Grant-in-Aid for Scientific Research (C) (19K08408). The other authors have no conflicts of interest to declare.

\section{Funding Sources}

This work was supported by an academic grant from JSPS KAKENHI (Grant No. JP21K15962).

\section{Author Contributions}

Y.H. and T.H. contributed to conception and design; acquisition of data was done by Y.H., J.T., M.I., Y.T., K.N., and S.Y.; analysis and interpretation of the data was done by Y.H. and N.I.; drafting of the article was take care of by Y.H.; critical revision of the article for important intellectual content was done by Y.H., T.H., N.I., J.T., M.I., Y.T., K.N., S.Y., A.I., T.Y., T.T., and J.F.; statistical analysis was done by Y.H. and N.I.; final approval of the article was done by Y.H., T.H., N.I., J.T., M.I., Y.T., K.N., S.Y., A.I., T.Y., and J.F.; and study supervision was done by T.H. and J.F.

\section{Data Availability Statement}

The data are not available for public access because of patient privacy concerns, but they are available from the corresponding author upon reasonable request.

Digestion 2022;103:159-168 


\section{References}

1 Torre LA, Bray F, Siegel RL, Ferlay J, LortetTieulent J, Jemal A. Global cancer statistics, 2012. CA Cancer J Clin. 2015 Mar;65(2):87108.

2 Bray F, Ferlay J, Soerjomataram I, Siegel RL, Torre LA, Jemal A. Global cancer statistics 2018: GLOBOCAN estimates of incidence and mortality worldwide for 36 cancers in 185 countries. CA Cancer J Clin. 2018 Nov;68(6): 394-424.

3 Ferro A, Peleteiro B, Malvezzi M, Bosetti C, Bertuccio P, Levi F, et al. Worldwide trends in gastric cancer mortality (1980-2011), with predictions to 2015, and incidence by subtype. Eur J Cancer. 2014 May;50(7):1330-44.

4 Allum WH, Blazeby JM, Griffin SM, Cunningham D, Jankowski JA, Wong $\mathrm{R}$, et al. Guidelines for the management of oesophageal and gastric cancer. Gut. 2011 Nov;60(11): 1449-72.

5 Thrumurthy SG, Chaudry MA, Hochhauser $\mathrm{D}$, Mughal M. The diagnosis and management of gastric cancer. BMJ. 2013 Nov 4;347: f6367.

6 Ezoe Y, Muto M, Uedo N, Doyama H, Yao K, Oda I, et al. Magnifying narrowband imaging is more accurate than conventional whitelight imaging in diagnosis of gastric mucosal cancer. Gastroenterology. 2011 Dec;141(6): 2017-25.

7 Yao K, Anagnostopoulos GK, Ragunath K. Magnifying endoscopy for diagnosing and delineating early gastric cancer. Endoscopy. 2009 May;41(5):462-7.

8 Sasajima K, Kudo SE, Inoue H, Takeuchi T, Kashida H, Hidaka E, et al. Real-time in vivo virtual histology of colorectal lesions when using the endocytoscopy system. Gastrointest Endosc. 2006 Jun;63(7):1010-7.

9 Kumagai Y, Monma K, Kawada K. Magnifying chromoendoscopy of the esophagus: invivo pathological diagnosis using an endocytoscopy system. Endoscopy. 2004 Jul;36(7): $590-4$.
10 Inoue H, Kazawa T, Sato Y, Satodate H, Sasajima K, Kudo SE, et al. In vivo observation of living cancer cells in the esophagus, stomach, and colon using catheter-type contact endoscope, "Endo-Cytoscopy system》. Gastrointest Endosc Clin N Am. 2004 Jul;14(3):58994.

11 Kumagai Y, Takubo K, Kawada K, Higashi M, Ishiguro T, Sobajima J, et al. A newly developed continuous zoom-focus endocytoscope. Endoscopy. 2017 Feb;49(2):176-80.

12 Sako T, Kudo SE, Miyachi H, Wakamura K, Igarashi K, Misawa M, et al. A novel ability of endocytoscopy to diagnose histological grade of differentiation in $\mathrm{T} 1$ colorectal carcinomas. Endoscopy. 2018 Jan;50(1):69-74.

13 Kaise M, Ohkura Y, Iizuka T, Kimura R, Nomura K, Kuribayashi Y, et al. Endocytoscopy is a promising modality with high diagnostic accuracy for gastric cancer. Endoscopy. 2015 Jan;47(1):19-25.

14 Tsurudome I, Miyahara R, Funasaka K, Furukawa K, Matsushita M, Yamamura T, et al. In vivo histological diagnosis for gastric cancer using endocytoscopy. World J Gastroenterol. 2017 Oct 7;23(37):6894-901.

15 Abad MRA, Shimamura Y, Fujiyoshi Y, Seewald S, Inoue H. Endocytoscopy: technology and clinical application in upper gastrointestinal tract. Transl Gastroenterol Hepatol. 2020 Apr;5:28.

16 Kudo SE, Misawa M, Wada Y, Nakamura H, Kataoka S, Maeda Y, et al. Endocytoscopic microvasculature evaluation is a reliable new diagnostic method for colorectal lesions (with video). Gastrointest Endosc. 2015 Nov;82(5): 912-23.

17 Horiuchi Y, Hirasawa T, Ishizuka N, Hatamori H, Ikenoyama Y, Tokura J, et al. Diagnostic performance in gastric cancer is higher using endocytoscopy with narrow-band imaging than using magnifying endoscopy with narrow-band imaging. Gastric Cancer. 2021 Mar; 24(2):417-27.
18 Muto M, Yao K, Kaise M, Kato M, Uedo N, Yagi K, et al. Magnifying endoscopy simple diagnostic algorithm for early gastric cancer (MESDA-G). Dig Endosc. 2016 May;28(4): 379-93.

19 Japanese Gastric Cancer Association. Japanese gastric cancer treatment guidelines 2018 (5th edition). Gastric Cancer. 2021 Jan;24(1): $1-21$.

20 Kataoka S, Kudo SE, Misawa M, Nakamura H, Takeda K, Toyoshima N, et al. Endocytoscopy with NBI has the potential to correctly diagnose diminutive colorectal polyps that are difficult to diagnose using conventional NBI. Endosc Int Open. 2020 Mar;8(3):E360-7.

21 Nakanishi H, Doyama H, Ishikawa H, Uedo N, Gotoda T, Kato M, et al. Evaluation of an e-learning system for diagnosis of gastric lesions using magnifying narrow-band imaging: a multicenter randomized controlled study. Endoscopy. 2017 Oct;49(10):957-67.

22 Yagi K, Nakamura A, Sekine A. Characteristic endoscopic and magnified endoscopic findings in the normal stomach without Helicobacter pylori infection. J Gastroenterol Hepatol. 2002 Jan;17(1):39-45.

23 Dixon MF, Genta RM, Yardley JH, Correa P. Classification, and grading of gastritis. The updated Sydney System. International workshop on the histopathology of gastritis, Houston 1994. Am J Surg Pathol. 1996 Oct;20(10): 1161-81.

24 Horiuchi Y, Fujisaki J, Yamamoto N, Shimizu T, Miyamoto Y, Tomida H, et al. Biological behavior of the intramucosal helicobacter pylori-negative undifferentiated-type early gastric cancer: comparison with helicobacter pylori-positive early gastric cancer. Gastric Cancer. 2016 Jan;19(1):160-5.

25 Horiuchi Y, Fujisaki J, Yamamoto N, Yoshimizu S, Ishiyama A, Yoshio T, et al. Diagnostic accuracy of demarcation using magnifying endoscopy with narrow-band imaging for helicobacter pylori-uninfected undifferentiated-type early gastric cancer. Gastric Cancer. 2018 Nov;21(6):988-97. 